\title{
Radioactive waste disposal
}

SIR - I have been following the debate on radioactive waste disposal for some time in Nature and elsewhere ${ }^{1}$. I accept that encasement and secure entombment is a viable means of disposing of high-level radioactive wastes, but the vexing problem of large volumes of medium- and low-level wastes remains. Deep slurry fracture injection into ancient geological sedimentary formations may represent an economic and socially acceptable means of executing this task.

For several years, we have been injecting large volumes of contaminated sand into porous, permeable, friable sandstone strata of Cretaceous age (Mannville Group, $>10^{8}$ million years BP) in Alberta and Saskatchewan. The sand is a waste product of heavy oil production ${ }^{2}$ and it is injected under pressures exceeding the overburden weight using an aqueous slurry, thereby fracturing it into the permeable sand. The aqueous phase dissipates by Darcian flow, and the sand remains in the near vicinity of the wellbore permanently entombed and immobile under the high stresses arising from the overburden weight.

In one case, still active, in excess of $12,000 \mathrm{~m}^{3}$ of sand has been injected into a depleted oil formation over a six-month period. Monitoring using adjacent wells and surface deformation field inversions demonstrated convincingly that the solid wastes are contained locally and that pressures dissipate rapidly. Injection depth is 400 metres, and environmental security is provided by a thick smectitic shale overburden (> 200 metres), flat-lying stratigraphy, absence of tectonic features and great lateral extent of the target strata. The interstitial water in these strata is older than $10^{7}$ years, and flow velocities are of the order of centimetres per year. Also, there are clay minerals dispersed in the sandstones, as well as in the adjacent shales, which should readily adsorb any polyvalent metal cations that leach from the wastes. We believe it is reasonable to speak in terms of million-year security for slurry fracture injection, given the proper geological conditions. Later this year, disposal of potash processing slimes (inert mineral matter) using the same approach will be undertaken with a comprehensive monitoring strategy.

Low- and medium-level, low-solubility solid radioactive wastes are excellent candidates for this type of disposal, and enhanced security could be achieved by adding a preponderance of shale fragments to the injected slurry, to provide local adsorption sites. Thus, given the right geological site (and many such sites exist), a granular solid waste can be prepared and injected with current petroleum industry technology at depths sufficient to entomb the wastes permanently, allowing the radioactive species to decay slowly. Our recent success with this leads us to suggest that it be considered for such materials, removing a barrier for the continued peaceful use of nuclear energy.

\section{Maurice B. Dusseault}

Porous Media Research Institute,

University of Waterloo,

Waterloo, Ontario, Canada N2L 3G1

1. Nature 375, 91-92 (1995).

2. Dusseault, M. B, Bilak, R. A., Bruno, M. S. Rothenburg, L. 1995. Disposal of Granular Solid Wastes in the Western Canadian Sedimentary Basin by Slurry Fracture Injection. Proceedings, Deep Well Injection Symposium Berkeley, California (in the press)

\section{Mannerly research}

SIR - No one would disagree with the basic sentiment expressed in your leading article (Nature 375, 4; 1995) that personal disputes should not interfere with scientific publishing, but in the particular case referred to we believe there may be misperceptions about what is considered as "good manners" in scientific research.

Six years ago, Professor Kenneth S. Kendler of Virginia Commonwealth University (VCU), together with staff from the Department of Mental Health at the Queen's University of Belfast and the Health Research Board in Dublin, began a study of the genetic epidemiology of schizophrenia in Ireland. Given the collaborative nature of this project, firm agreements were established at the outset about ownership of the data collected and authorship of any future publications. It would have been impossible to undertake this type of project without this explicit understanding and mutual trust. Throughout this study, we, the authors of this letter, have been fully involved in discussions with Kendler about future directions and possible publications relating to the project. One of us was involved in a site visit by members of the US National Institute for Mental Health to VCU which led to an extension of the funding of the study.

Dr Scott R. Diehl was an important part of this collaboration until two years ago when he left the laboratory at VCU. After his departure we were aware of the continuing dispute between Diehl and Kendler. However, during this time Diehl has had no communication with us either about his role in the project or the use of the data collected. We were therefore unaware of recent publication based on this subject until a few days before it appeared in Nature Genetics. We find it unacceptable that this publication did not properly acknowledge the other institutions involved or the collaborative nature of the study. It is unclear from the published paper whether Diehl has in fact taken data from VCU without the agree- ment of the collaborating partners.

By contrast, Diehl was kept aware of the continuing work on the chromosome $6 p$ region in the laboratory at VCU and was given a copy of the manuscript that was to be submitted and in which Diehl's role in the original finding was acknowledged.

It is therefore difficult for us to accept that "no one behaved wrongly"; indeed we believe that the issues involved go far beyond those of "priority" in scientific publications.

Finally, borrowing on your metaphor, one member of the original partnership group did publish with the author list chopped in half. We wonder what Solomon's judgement would have been in this case.

\section{R. J. McClelland}

F. A. O'Neill

F. J. Duke

School of Clinical Medicine,

The Queen's University of Belfast,

Belfast BT7 1NF, UK

\section{Dundee did well}

SIR - Ehsan Masood (Nature 375, 96; 1995) correctly pointed out that the University of Leicester's biochemistry department overtook Dundee in terms of research income per full-time academic according to the CVCP/UFC Management Statistics for 1993-94. Despite some anomalies in the calculations (for example establishing an MRC unit removed a considerable source of income from our return), Dundee came a close second, ahead of both Oxford and Imperial College and not, as the article implies, somewhere among the also rans.

\section{P. Downes}

Biochemistry Department,

University of Dundee,

Dundee DD1 4HN, UK

\section{Earlier than that}

SIR - John Maddox (Nature 374, 759; 1995 ) is incorrect in stating, with regard to the success of Einstein's theory of general relativity, that: "The bending of light near the limb of the Sun at the solar eclipse of 1919 was the first validation .... The precession of the perihelion of Mercury came later."

According to Steven Weinberg (Gravitation and Cosmology, page 12, Wiley, New York, 1972), the precession of the perihelion of Mercury was discovered by LeVerrier even before LeVerrier predicted the existence of Neptune. The perihelion precession was confirmed in 1882 by Simon Newcomb.

Richard C. Henry

Department of Physics and Astronomy, Johns Hopkins University,

Baltimore, Maryland 21218-2695, USA 